\title{
Demanda de información y conocimiento de los pacientes de su derecho a decidir o rechazar una cirugía. Experiencia en dos servicios quirúrgicos
}

\author{
Fernanda Lozano*, Laura Borgno ${ }^{\dagger}$, Franco González Mora ${ }^{\ddagger}$, Fidel Lagos§, \\ Frances Borches§, Patricia Wetsch", Gabriel Barrero** , Hugo Rodríguez Almadat†
}

\section{Resumen}

El consentimiento informado como deber del médico y derecho de los pacientes está expresamente reconocido en la legislación nacional con especial énfasis a partir de la aprobación de la Ley Nº 18.335 en el año 2008. Sin embargo, se han evidenciado dificultades en el proceso de información y obtención del consentimiento. Mediante un cuestionario cerrado, anónimo y autoadministrado se consultó a pacientes operados sobre qué aspectos de la información consideraban relevantes conocer y qué conocimiento tenían sobre el derecho a ser informados. Más del $80 \%$ de los entrevistados manifestó el interés en contar con información sobre alternativas terapéuticas y sobre las complicaciones más frecuentes de la intervención. Asimismo, creyeron pertinente contar con la descripción del procedimiento al que serán sometidos y las opciones de modificar el tipo de cirugía durante la operación. Menos del 10\% explicitó el interés en ser informado sobre el riesgo de muerte, la posibilidad de reintervenciones y en recibir información mediante figuras e imágenes ilustrativas del procedimiento. Se destaca que nueve de cada diez entrevistados admitieron conocer su derecho a ser informados sobre diferentes aspectos del acto quirúrgico y que casi la mitad (48\%) consideró que debe confiar en las decisiones del médico sin importar la cantidad de información recibida.

Palabras clave: CONSENTIMIENTO INFORMADO

CIRUGÍA

DERECHOS DEL PACIENTE

ACCESO A LA INFORMACIÓN

Key words: INFORMED CONSENT

SURGERY

PATIENT RIGHTS

ACCESS TO INFORMATION

\footnotetext{
* Profesora Adjunta del Departamento de Medicina y Ciencias Forenses. Facultad de Medicina. Universidad de la República. Uruguay. † Profesora Adjunta de la Clínica Quirúrgica 2. Facultad de Medicina. Universidad de la República. Uruguay.

¥ Profesor Adjunto de la Unidad de Sociología de la Salud, Departamento de Medicina Preventiva y Social. Facultad de Medicina. Universidad de la República. Uruguay.

$\S$ Asistente del Departamento de Medicina Legal y Ciencias Forenses. Facultad de Medicina. Universidad de la República. Uruguay. II Posgrado del Departamento de Medicina Legal y Ciencias Forenses. Facultad de Medicina. Universidad de la República. Uruguay.

${ }^{* *}$ Asistente de la Unidad de Sociología de la Salud, Departamento de Medicina Preventiva y Social. Facultad de Medicina. Universidad de la República. Uruguay.

†† Profesor Director del Departamento de Medicina Legal y Ciencias Forenses. Facultad de Medicina. Universidad de la República. Uruguay. Los autores declaran no tener conflicto de intereses.

Correspondencia: Dra. Fernanda Lozano. Avda. Gral. Flores 2125. Montevideo, Uruguay. Correo electrónico: fernandalozano@vera.com.uy
} 


\section{Introducción}

El consentimiento informado en medicina supone un proceso de diálogo por el cual el paciente obtiene información en relación con la enfermedad que le fue diagnosticada, la naturaleza del procedimiento terapéutico, los riesgos, beneficios, alternativas y pronósticos ${ }^{(1)}$. Es esencialmente un acto de comunicación que se desarrolla en escenarios que implican relaciones de poder asimétricas entre usuarios y profesionales de la medicina. Contribuyen a configurar estos escenarios las instituciones, los marcos jurídicos, el tipo de actores y saberes involucrados, así como las emociones y subjetividades de todos los actores (pacientes, familiares, personal médico y no médico).

El proceso de obtención del consentimiento informado forma parte del acto médico, siendo un derecho de los pacientes y un deber del médico ${ }^{(2)}$ expresamente reconocido en la legislación nacional con especial énfasis a partir de la aprobación de la Ley $\mathrm{N}^{\circ} 18.335$ en 2008 y su reglamentación (Decreto 274/10). La obtención del consentimiento informado es un componente del cumplimiento de la lex artis ad hoc del acto médico y un aspecto crítico de la responsabilidad profesio$\mathrm{nal}^{(3)}$. Otras leyes abordan el consentimiento informado desde un punto de vista general (Ley $\mathrm{N}^{\circ} 19.286$ ) y para situaciones especiales (leyes 14.005, 18.426, $18.473,18.987$ y 19.519$)$.

El consentimiento informado es válido cuando resulta de una decisión de un paciente libre de coacción y presiones externas, suficientemente informado, competente para comprender la información y decidir de acuerdo a sus propios valores. Debe ser solicitado en forma oportuna y en función al tipo de procedimiento y estado del paciente ${ }^{(2-4)}$. De esta forma, la regla de consentimiento se basa en el reconocimiento a la autonomía y libertad de los sujetos para tomar decisiones sobre sí mismos y en particular sobre su cuerpo en función de sus valores, intereses y proyecto de vida ${ }^{(5)}$.

Sin embargo, es necesario advertir la importancia que adquieren las relaciones de poder asimétricas que atraviesan el vínculo médico-paciente, que suele incluir asimetría de saberes y vulnerabilidad y fragilidad emocional del paciente ${ }^{(5)}$. Boltansky hace referencia a la asimetría propia de la relación médico-paciente en un marco de distancia social entre clases. Cuando se trata de personas de clases populares, el médico limita la transmisión de saberes. Se siente dispensado de explicarle al paciente. El paciente luego reproduce, reinterpreta y reduce las categorías propias de la medicina a categorías generales y familiares del lenguaje. A mayor distancia social médico-enfermo, menor capacidad del enfermo para memorizar y reproducir los términos científicos. El autor señala que “...cuanto mayores sean las diferencias entre el sistema de categorías utilizado por el médico y el sistema utilizado por el paciente, tanto más difícil será el 'trabajo' de reinterpretación o de 'reinvención' que exigirá la asimilación por parte del enfermo del discurso fragmentado, hermético y cifrado del médico, que implica su 'comprensión y 'memorización ${ }^{\prime(6)}$."

De acuerdo a Galán Cortés, "el consentimiento del paciente se extenderá, en cuando a su validez y eficacia, hasta donde haya sido informado"(7). Pero la calidad y cantidad de información a brindar suele generar dudas al equipo asistencial. El consentimiento informado, si bien implica un acto comunicativo verbal, debe ser registrado en la historia clínica como todo acto médico ${ }^{(3,5)}$. La normativa exige, además, que en caso de procedimientos invasivos e intervenciones quirúrgicas deberá otorgarse por escrito (Decreto 274/010). La entrega de información por escrito puede ser de gran utilidad para habilitar el proceso de reflexión y diálogo, clarificando dudas de la información que previamente se debió brindar en forma verbal y a la que no puede sustituir ${ }^{(2,8)}$.

En 1995, Bespali y colaboradores evaluaron la percepción de los pacientes en relación a quién tiene que tomar la decisión frente a los tratamientos quirúrgicos y médicos. Se observó que las respuestas de los pacientes fueron diferentes según el tipo de tratamiento propuesto (quirúrgico o médico) y que le asignaron distinto grado de participación a su familia en la toma de estas decisiones ${ }^{(9)}$.

González y colaboradores estudiaron en 2010 la cantidad y calidad de la información conocida por pacientes que consintieron cirugías de coordinación. De este estudio se desprende que una porción considerable de pacientes no recibió, no recordó o no pudo verbalizar información relevante para la toma de decisiones, aun habiendo autorizado por escrito el procedimiento quirúrgico. La investigación concluyó que es necesario profundizar en el conocimiento de las debilidades existentes a la hora de informar y mejorar el proceso como forma de proteger los derechos de los pacientes y de practicar la cirugía con mayor seguridad jurídica ${ }^{(10)}$. Asimismo, otros estudios llevados a cabo en el país han señalado fallas en el proceso de información y obtención del consentimiento, así como su registro ${ }^{(11,12)}$.

El presente estudio tuvo como propósito evaluar los aspectos relacionados a la información que los pacientes internados por patología quirúrgica consideran relevantes conocer, así como el nivel de conocimiento que tienen sobre su derecho a ser informados.

\section{Población y método}

Se realizó una encuesta a pacientes internados en servicios quirúrgicos en los hospitales de Las Piedras y Ma- 
ciel (Clínica Quirúrgica 2) en el período comprendido entre el 13 de julio y el 16 de agosto de 2015 .

\section{Criterios de inclusión}

a) Estar internado en sala de cirugía del Hospital de Las Piedras o en la Clínica Quirúrgica 2 del Hospital Maciel.

b) Haber sido intervenido dentro de los siete días previos a la recogida de datos.

c) Haber estado en sala al momento de realizarse la encuesta.

d) Haber prestado su consentimiento informado para participar en la investigación.

\section{Criterios de exclusión}

a) Portadores de patologías neuro-psiquiátricas o incapaces de leer la encuesta.

b) Pacientes que por las características del posoperatorio no estaban en condiciones de ser entrevistados (por ejemplo, dolor, incomodidad, cursando un posoperatorio tórpido con vómitos, uso de sonda nasogástrica).

c) Pacientes que por su patología de base requirieron la participación de otras especialidades quirúrgicas (urología, cirugía vascular, cirugía de tórax).

El instrumento empleado fue un cuestionario cerrado, anónimo, autoadministrado y de carácter voluntario elaborado por el equipo de investigadores (ver anexo). Fue aplicado por integrantes del equipo que no estaban involucrados en la relación clínica con los pacientes encuestados.

Para la generación de la base de datos se empleó el programa EpiData v 3.1. El procesamiento estadístico se realizó mediante el paquete estadístico SPSS versión 18. Los gráficos se generaron en MsExcel 2010. Se realizó un análisis descriptivo bivariado con distribuciones porcentuales y absolutas expresadas en tablas de doble entrada.

El protocolo de investigación fue aprobado por el Comité de Ética de ambos hospitales. Se informó a los participantes los objetivos, alcance del estudio, manejo anónimo de los datos, derecho a negarse a participar sin que su decisión afectara la calidad de la atención y se les solicitó el consentimiento informado para participar. La información individual fue manejada en forma confidencial y en exclusividad por los integrantes del equipo, no se empleó con otra finalidad más que para determinar los criterios de inclusión o exclusión.

\section{Resultados}

En el período en que se llevó a cabo el relevamiento hubo un total de 111 cirugías de coordinación ( 38 de las cuales fueron en la Clínica Quirúrgica 2 del Hospital Maciel y 24 en el Hospital de Las Piedras) y 103 cirugías de urgencia/emergencia (43 que ingresaron a la Clínica 2 del Hospital Maciel y 17 en el Hospital de Las Piedras). De esos, un total de 31 pacientes cumplieron con los criterios de inclusión y no tenían criterios de exclusión. Se destaca que cinco pacientes no prestaron su consentimiento informado para participar en la investigación.

Las dimensiones analizadas fueron las siguientes:

1. Demandas de información.

2. Conocimiento del derecho a la información.

3. Proceso de información para la intervención.

Dimensión 1 - Demandas de información. Se interrogó en relación con qué aspectos del procedimiento deseaban conocer, cuáles deseaban ignorar y cuáles les resultaban indiferentes.

Se constata que un grupo mayoritario de entrevistados demanda que se les informe sobre todos y cada uno de los aspectos indagados (figura 1). En cuatro de estos ítems se constata un alto interés (mayor a $80 \%$ ) por acceder a mayor conocimiento; los niveles de rechazo a conocer fueron muy bajos (inferiores a 10\%).

En un segundo lugar, con niveles más bajos de deseo de conocimiento, están las complicaciones de mayor gravedad (74\%), el riesgo de muerte (74\%), posibilidad de reintervenciones (68\%), utilización de figuras o esquemas para describir la técnica operatoria o el tipo de incisión (52\%) (figura 1). En estos ítems existieron niveles relativamente elevados de rechazo e indiferencia a la información.

Dimensión 2 - Conocimiento del derecho a la información. En primer lugar se relevó el conocimiento del derecho a la información sobre aspectos de la cirugía requeridos por la normativa vigente (Decreto 274/010): diagnóstico de la enfermedad, tratamientos necesarios para la curación, calidad y grado académico del cirujano actuante, estudios necesarios para el diagnóstico de la enfermedad y la valoración preoperatoria, complicaciones durante la cirugía, pronóstico de la enfermedad y posibles tratamientos alternativos para evitar la cirugía. Los resultados se detallan en la figura 2.

En segundo lugar se valoró si estaban de acuerdo o en desacuerdo con distintas frases referentes a derechos de información, donde esos derechos son puestos en cuestión (figura 3): obligación del médico de responder todas las dudas acerca de la cirugía y complicaciones; si a pesar de la firma del formulario de consentimiento informado por el paciente, el médico sigue siendo responsable de eventuales complicaciones de la cirugía; si tie- 


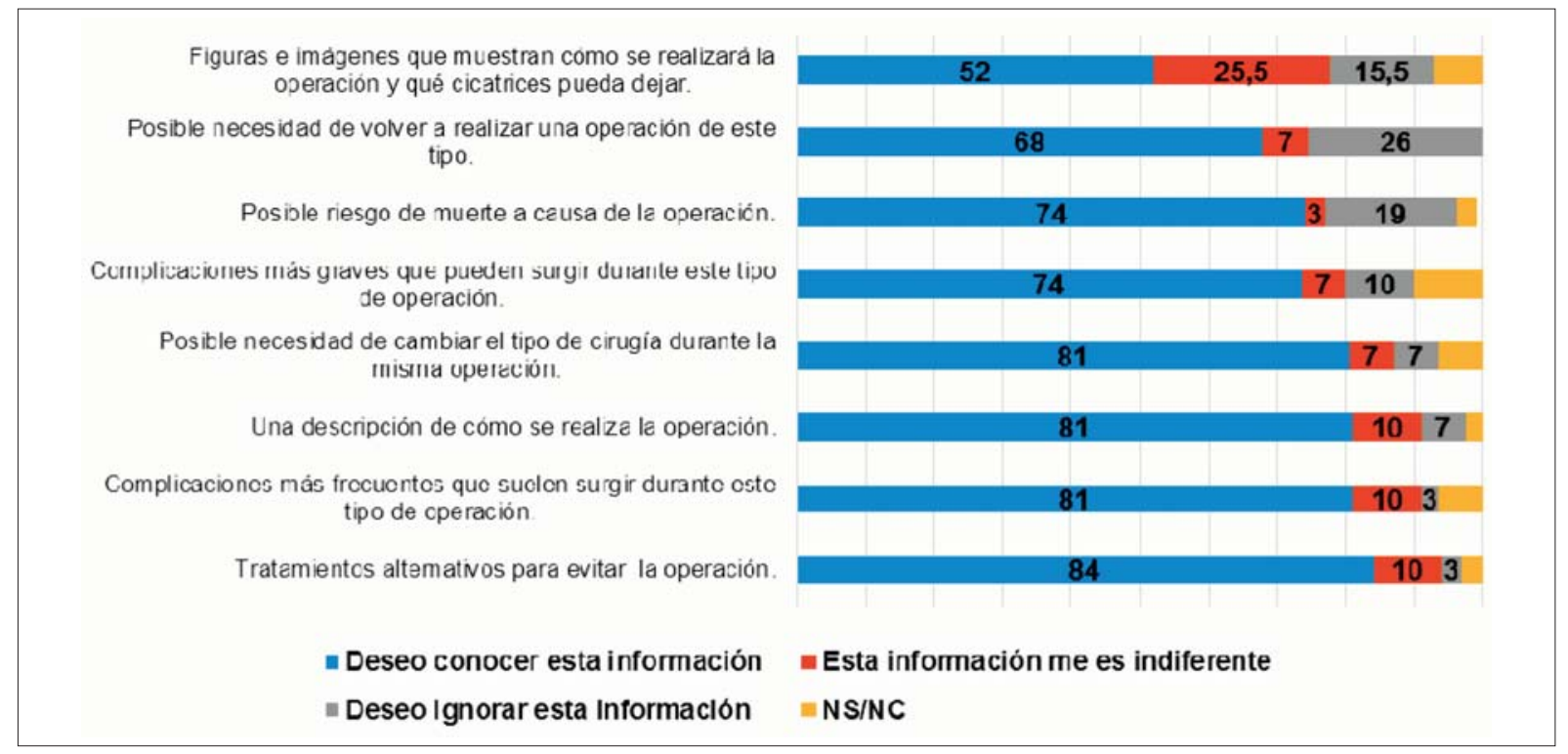

Figura 1. Demandas de información

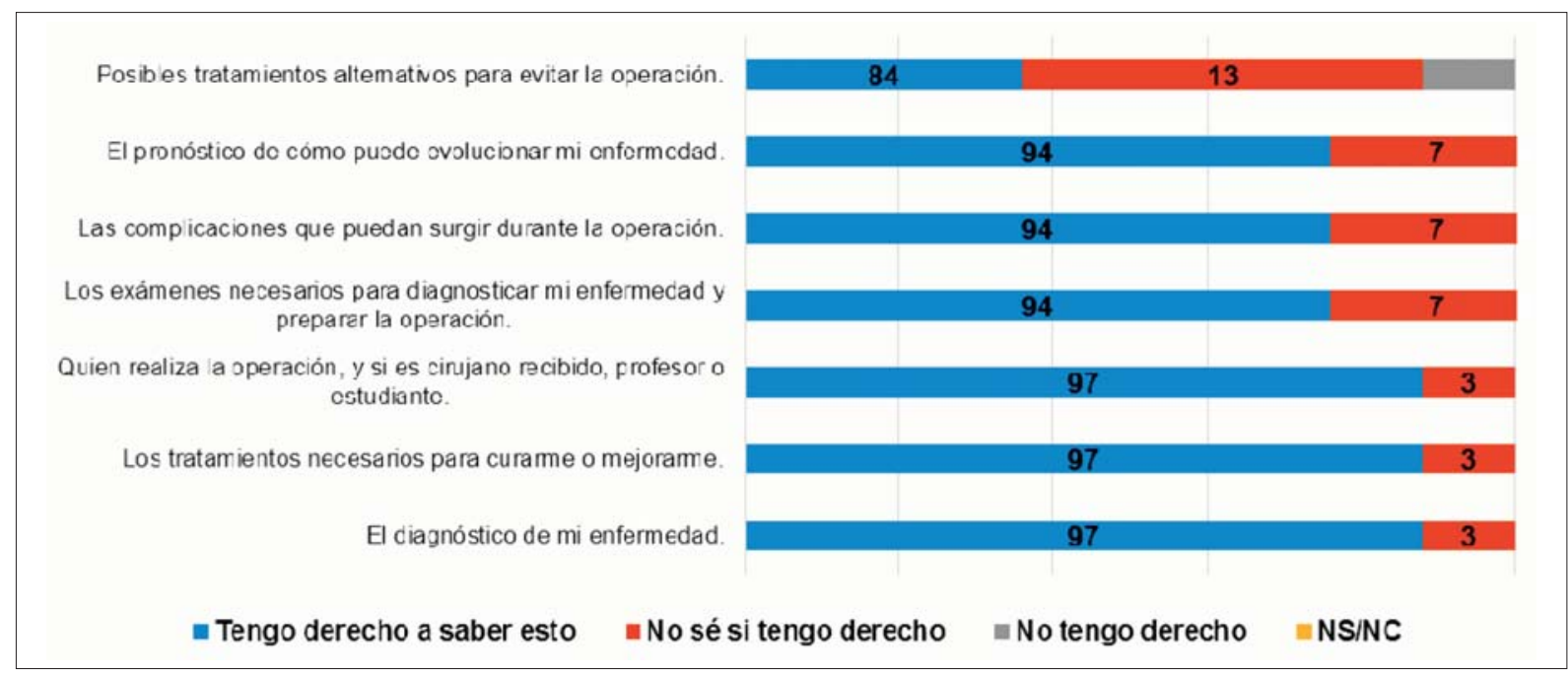

Figura 2. Conocimientos de derechos de información sobre aspectos de la operación

ne derecho a negarse a ser interrogado y examinado por estudiantes y que su caso sea usado con fines docentes; si siempre debe confiar en decisiones del médico sin importar el volumen de información brindada.

Existió un nivel de acuerdo del $90 \%$ con la idea de que el médico responsable de la operación está obligado a responder todas las dudas que tiene el paciente respecto a la operación y sus consecuencias. Respecto de los alcances legales del consentimiento informado, el 23\% no sabía que el consentimiento no quita responsabilidad legal al médico en un caso de mala praxis médica y $62 \%$ no conocía su derecho a anular el consentimiento en cualquier momento luego de firmado. Solo $55 \%$ de los pacientes conocía su derecho a no saber diversos aspectos de su operación.

\section{Discusión}

Hace dos décadas un estudio ${ }^{(9)}$ tendiente a evaluar el conocimiento que tenían las personas de sus derechos como pacientes mostró que solo cuatro de cada diez exigían espontáneamente información relevante para brindar su consentimiento o rechazar un tratamiento. No obstante, dada la metodología diferente empleada en esa investigación y la disparidad entre las poblaciones estudiadas, no resulta comparable con el estudio de Bespali, en que el grupo de pacientes encuestados mos- 


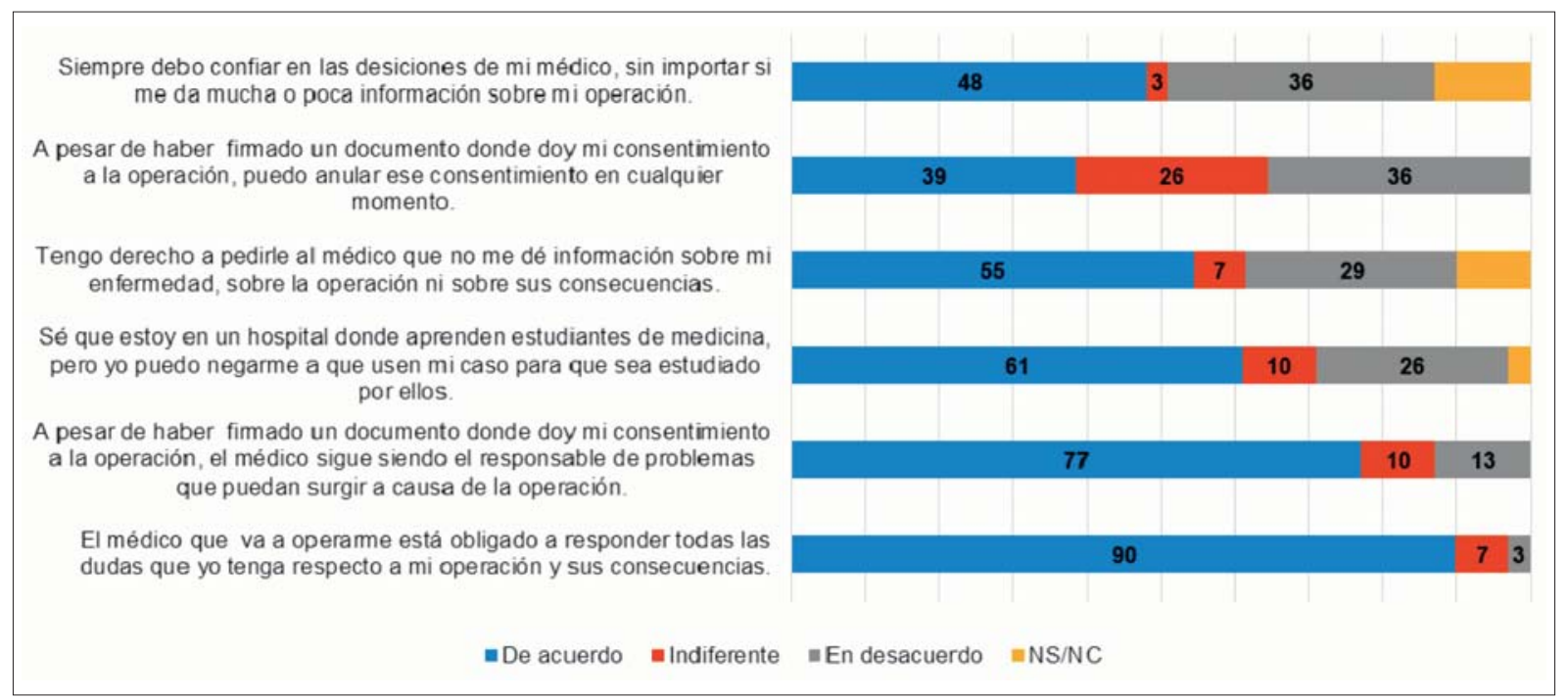

Figura 3. Concordancia con frases sobre ejercicio de información

tró un nivel de exigencia elevado en relación con la información necesaria.

En el grupo de pacientes encuestados se encontraron diferencias entre los aspectos que preferentemente se desean conocer. El 80\% de los entrevistados desea información sobre cuatro aspectos fundamentales vinculados al tratamiento de su enfermedad: tratamientos alternativos a la operación, complicaciones más frecuentes, descripción del procedimiento quirúrgico y su modificación durante la cirugía. Por el contrario, las complicaciones de mayor gravedad, el riesgo de muerte, la posibilidad de una reintervención quirúrgica, presentan menor deseo de conocimiento.

Estos datos pueden interpretarse en términos de la existencia de una estructura de demandas de información pragmáticamente delineada. En primer lugar se buscaría conocer la posibilidad de evitar un tratamiento quirúrgico mediante otros tratamientos alternativos. En segundo lugar se intentaría acceder al conocimiento básico sobre las complicaciones más frecuentes de la intervención, el procedimiento de la intervención, así como sobre la posibilidad de tener que modificar ese procedimiento en el mismo momento de la operación. En tercer lugar aparece el deseo de conocer episodios de alto nivel de gravedad (complicaciones más graves y peligro de muerte), que si bien son de gran interés pasan a un segundo plano como consecuencias no deseadas de un procedimiento cuya inevitabilidad ya se confirmó y cuya probabilidad de ocurrencia es relativamente menor, $o$ bien por mecanismos de negación. En último lugar aparece el deseo de conocimiento de la posibilidad de una reintervención quirúrgica (que podría ser considerado como un tema irrelevante hasta no conocer el resultado de la primera operación), así como lo estético, que quedaría relegado al último nivel de relevancia como aspecto a informar al contraponerlo con aspectos tales como la posibilidad de perder la vida o sufrir una incapacidad permanente.

Por otro lado, la mitad de los pacientes encuestados indicó que deseaba conocer, mediante figuras o esquemas, la técnica operatoria e incisión utilizada, lo que justificaría la elaboración de nuevos formularios de consentimiento informado específicos e ilustrativos. Con una sola excepción los encuestados coincidieron en que deseaban conocer el diagnóstico de su enfermedad, los tratamientos necesarios y quién realizaría la operación. Solamente dos de los encuestados no manifestaron interés en saber cuáles son los exámenes necesarios para el diagnóstico y valoración de su enfermedad, las complicaciones que pueden surgir en la cirugía y el pronóstico o evolución de la enfermedad.

Cuando se evaluó el conocimiento del derecho a la información en relación con diferentes aspectos de la cirugía, para cada atributo se constataron altísimos niveles de conocimiento de derechos. Entre $84 \%$ y $97 \%$ de los pacientes refirió conocer su derecho a ser informado respecto a los puntos que establece la normativa vigente, entre ellos el diagnóstico de la enfermedad, tratamientos necesarios para la curación o mejoría de la enfermedad, conocimiento acerca de la calidad y grado académico del cirujano actuante, los estudios diagnósticos necesarios para el diagnóstico de la enfermedad y para la valoración preoperatoria, las complicaciones durante la cirugía, el pronóstico de la enfermedad, los posibles tratamientos alternativos para evitar la cirugía. Llama la atención que el único aspecto sobre el cual aparece ma- 
yor nivel de dudas acerca de constituirse como un derecho (tratamientos alternativos para evitar la cirugía), con $84 \%$ de conocimiento del derecho, sea justamente aquel aspecto sobre el cual existe una demanda más prioritaria de información: uno de cada diez pacientes desconoce que tiene derecho a ser informado sobre eventuales tratamientos alternativos a la operación, a pesar de ser este el aspecto del cual existe una demanda más prioritaria de información. Este aspecto debería ser tenido en cuenta para facilitar que el paciente ejerza su derecho de autonomía y toma de decisiones libremente, contando con todas las opciones disponibles. En la evaluación de concordancia con frases sobre ejercicio del derecho de información, los resultados mostraron nuevamente elevados porcentajes de conocimiento del derecho a que sus inquietudes sean evacuadas, ya que aparece un nivel de acuerdo muy alto (90\%) con la idea de que "el médico que va a operarme está obligado a responder todas las dudas que yo tenga respecto a mi operación y sus consecuencias". Esto refuerza la idea de que los pacientes conocen su derecho de acceder a toda la información que consideren necesaria respecto de las características de la operación.

Los pacientes de nuestra muestra mostraron un conocimiento elevado respecto a sus derechos a ser informados. Sin embargo, cuando se indagó el nivel de conocimiento de los derechos efectivos y especialmente sobre los alcances médico-legales del consentimiento informado, más de la mitad de los pacientes desconocía su derecho a revocar un consentimiento informado otorgado por escrito y firmado previamente (72\%).

Dos dimensiones adicionales hacen referencia al conocimiento de derechos acerca de la forma cómo se produce la relación médico-paciente. En primer término, cuatro de cada diez entrevistados desconocían la posibilidad de negarse a que su caso sea tomado como motivo de estudio por parte de estudiantes de Medicina. En segundo lugar, casi la mitad de los pacientes encuestados (48\%) manifestó acuerdo con que "Siempre debo confiar en las decisiones de mi médico, sin importar si me da mucha o poca información sobre mi operación", y 30\% de los pacientes desconoce su derecho a negarse a recibir información sobre la enfermedad y el tratamiento.

Es significativo que con el nivel de conocimiento de los derechos expresados por los pacientes casi la mitad refiere que siempre debe confiar en las decisiones del médico, sin importar el volumen de la información, lo que puede traducir la coexistencia de dos modelos en la relación médico-paciente: el paternalista, que prioriza el lugar del médico en función del principio de beneficencia, y el autonomista, que jerarquiza la libertad del paciente a decidir sobre su cuerpo. Este fenómeno ha sido denominado "transición bioética"(5,13).
El comportamiento de estos últimos indicadores, así como las opiniones sobre el alcance legal del consentimiento informado, ponen de relieve una situación donde, si bien los pacientes parecen tener pleno conocimiento de su derecho a la información, no conocen cabalmente la base legal, institucional y relacional con que cuentan para hacer valer esos derechos.

Como limitación del estudio se destaca que se presenta una descripción univariada de las dimensiones relevadas debido a que el número de casos totales (31 pacientes) de esta fase preliminar no permite realizar aperturas, según variables de segmentación, sin afectar la distribución normal del error aleatorio en la medición de sus parámetros. Un mayor conocimiento de estos aspectos requeriría nuevos estudios.

\section{Conclusiones}

El grupo de pacientes encuestados mostró un nivel elevado de conocimiento de su derecho a la información, constatándose altos niveles de demanda de información referente a la patología, el procedimiento quirúrgico y las alternativas terapéuticas. Este conocimiento de sus derechos es más restringido cuando se indagó específicamente sobre determinados aspectos referentes al alcance médico-legal del consentimiento informado, como la posibilidad de revocar su decisión.

Resulta significativo que un porcentaje considerable de los pacientes crea que el consentimiento informado exime de responsabilidad legal al médico y que la mayoría ignorara su derecho a rectificar el consentimiento informado.

Sigue siendo un desafío lograr que todas las personas, independientemente de su origen social y entorno cultural, puedan tomar decisiones sobre sí mismos y en particular sobre su cuerpo en función de sus valores, intereses y proyecto de vida.

\section{Summary}

Informed consent as the physician's obligation and the patient's right is expressly recognized by the national law, mainly as from 2008, when Act Number 18335 was passed. However, certain difficulties have been seen in the information process and in connection with obtaining consent. Patients who underwent surgery were consulted by means of a closed, anonymous and self-administered survey as to the specific aspects they considered relevant to know and how familiar they were with their right to be informed. Over $80 \%$ of the patients in the survey stated they were interested in learning about treatment alternatives and the most frequent complications arising from treatment. Likewise, they thought it would be appropriate to know about the procedure they would undergo and the options available to 
modify the kind of surgery once it had started. Under 105 of patients stated they were interested in learning about life-threatening risks, the chance of a needing to operate again and receiving information through pictures and illustrations showing the procedure. The study points out 9 out of ten patients admitted they knew about their right to be informed about different aspect of the surgery and that almost half of them (48\%) stated they needed to trust the decisions made by physicians, regardless of the quality of the information received.

\section{Resumo}

O consentimento informado, como dever do médico e direito dos pacientes, está expressamente reconhecido na legislação nacional com especial ênfase a partir da aprovação da Lei $N^{\circ} 18.335$ de 2008 . No entanto foram observadas dificuldades no processo de informação e obtenção do consentimento. Um grupo de pacientes operados foi consultado sobre que aspectos da informação consideravam relevantes conhecer e que conhecimento tinha sobre o direito a ser informados utilizando um questionário fechado, anônimo e auto-administrado. Mais de $80 \%$ dos entrevistados mostrou interesse em contar com informação sobre alternativas terapêuticas e sobre as complicações mais frequentes da cirurgia. Ademais, acharam importante contar com a descrição do procedimento ao que seriam submetidos e as opções de modificar o tipo de cirurgia durante a operação. Menos de $10 \%$ mostrou interesse em ser informado sobre o risco de morte, a possibilidade de ser reoperado e em receber informação através de figuras e imagens ilustrativas do procedimento. Destaca-se que nove de cada dez entrevistados admitiu conhecer seu direito a ser informado sobre diferentes aspectos da cirurgia e que quase a metade (48\%) considerou que devia confiar nas decisões do médico sem importar a quantidade de informação recebida.

\section{Bibliografía}

1. Tena Tamayo C. El consentimiento informado en la práctica médica. Una visión humanista. Rev CONAMED 2004; 9(3):8-10.

2. Galán Cortés J. La responsabilidad médica y el consentimiento informado. Rev Méd Urug 1999; 15:5-12.

3. Rodríguez Almada H. Los aspectos críticos de la responsabilidad médica y su prevención. Rev Méd Urug 2001; 17(1):17-23.

4. Comisión Nacional de Arbitraje Médico. Consentimiento válidamente informado. México D.F.: CONAMED, 2004.

5. Rodríguez Almada H. Consentimiento informado en la práctica clínica. AnaFaMed 2017; 4(Sup 2):22-30.

6. Boltanski L. Los usos sociales del cuerpo. Bs As: Periferia, 1975: 27.

7. Galán Cortés J. El consentimiento informado. En: Aspectos legales de la relación clínica. Madrid: Jarpyo, 2000: 27-38.

8. Simón Lorda P, Júdez Gutiérrez J. Consentimiento informado. Med Clín 2001; 117(3):99-106.

9. Bespali Y, de Pena M, Consens M. Derechos de los pacientes en Uruguay: percepción de los usuarios de hospitales. Rev Méd Urug 1995; 11:81-98.

10. González D, Rodríguez Almada H, Ruso L. Cantidad y calidad de la información conocida por pacientes que consintieron cirugías de coordinación. Rev Méd Urug 2010; 26:25-31.

11. González González D, Rodríguez Almada H, Berro Rovira G. Consentimiento informado: análisis crítico de su aplicación en un servicio quirúrgico. Rev Méd Urug 2005; 21(4):291-7.

12. Rodríguez Almada H. Seudoconsentimiento informado en Uruguay. Rev Méd Urug 2002; 18(1):89-93.

13. Rodríguez Almada H. Derechos de los pacientes en Uruguay: normativa vigente y organizaciones de usuarios. En: Memorias del V Simposio Internacional CONAMED. México DF: CONAMED, 2000:137-43. 
Anexo. Cuestionario
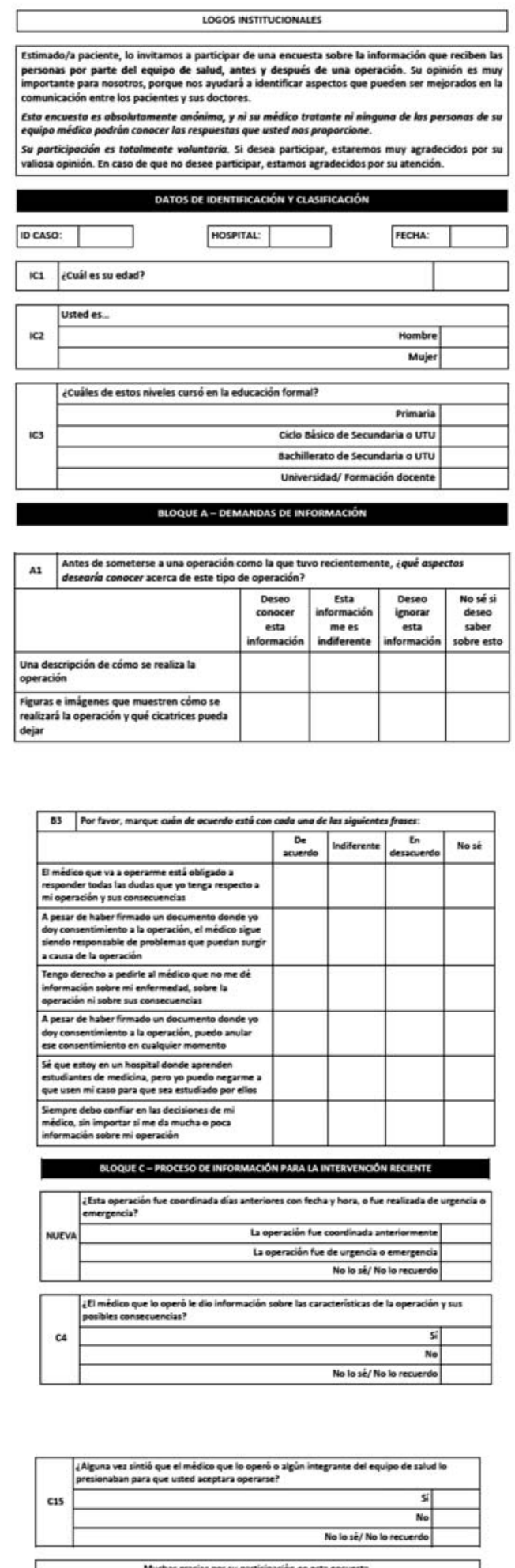

Muchas gracias par su participacion en etts encuets.

\begin{tabular}{|c|c|c|c|c|}
\hline & $\begin{array}{l}\text { Deseo } \\
\text { conocer } \\
\text { esta } \\
\text { información }\end{array}$ & $\begin{array}{l}\text { fots } \\
\text { infformacion } \\
\text { me es } \\
\text { indiferente }\end{array}$ & 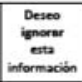 & $\begin{array}{c}\text { No sé si } \\
\text { deseo } \\
\text { saber } \\
\text { sobre etoto }\end{array}$ \\
\hline \multicolumn{5}{|l|}{$\begin{array}{l}\text { Posibie riestgo de muerte a causa de la } \\
\text { operaón }\end{array}$} \\
\hline \multicolumn{5}{|l|}{$\begin{array}{l}\text { Complicaciones mis frecuentes que suelen } \\
\text { surgir durante eate tipo de opersión }\end{array}$} \\
\hline \multicolumn{5}{|l|}{$\begin{array}{l}\text { Complicaciones mbis graves que pueden } \\
\text { surgir durante este tipo de operaión }\end{array}$} \\
\hline \multicolumn{5}{|l|}{$\begin{array}{l}\text { Tratamientos athernativos pars evitar to } \\
\text { opersobin }\end{array}$} \\
\hline \multicolumn{5}{|l|}{$\begin{array}{l}\text { Posabie necesided de volver a realitar una } \\
\text { opercón de este tipo }\end{array}$} \\
\hline 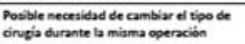 & & & & \\
\hline
\end{tabular}

BLOQUE B - DERECHOS DE INFORMACCON

\begin{tabular}{|c|c|c|c|c|}
\hline \multirow[t]{2}{*}{82} & \multicolumn{4}{|c|}{ 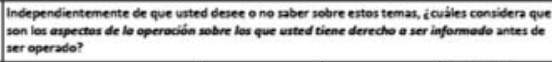 } \\
\hline & & $\begin{array}{l}\text { Tenso derecho a } \\
\text { saber esto }\end{array}$ & \begin{tabular}{|l|} 
No tengo derectho \\
a saber esto
\end{tabular} & $\begin{array}{l}\text { No sé si tengo } \\
\text { derecto a saber } \\
\text { evto }\end{array}$ \\
\hline \multicolumn{5}{|c|}{ El diagnéstico de mi entermedad } \\
\hline \multicolumn{5}{|c|}{ 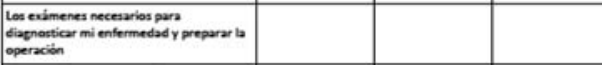 } \\
\hline \multicolumn{5}{|c|}{$\begin{array}{l}\text { los tratamientes necetarios para curarme } \\
\text { o mejorame }\end{array}$} \\
\hline \multicolumn{5}{|c|}{$\begin{array}{l}\text { Las complicaciones que puedan surgir } \\
\text { durnte la operación }\end{array}$} \\
\hline \multicolumn{5}{|c|}{$\begin{array}{l}1 \text { pronóstico de cómo puede evoludionar } \\
\text { mi entermedad }\end{array}$} \\
\hline \multicolumn{5}{|c|}{$\begin{array}{l}\text { Posibles tratamientos aternativos para } \\
\text { evitar la operación }\end{array}$} \\
\hline $\begin{array}{l}\text { Quitin } \\
\text { reotid }\end{array}$ & 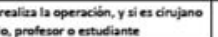 & & & \\
\hline
\end{tabular}

